\title{
The DPP-4 inhibitor sitagliptin alters the gut microbiota and prevents neuronal damage of hippocampus in STZ induced type 2 diabetic rats
}

\section{Ying Chang}

Chongqing General Hospital,University of Chinese Academy of Sciences

\section{Ting Xiang}

The Second hospital of Chongqing medical university

\section{Hongyan Wang}

Chongqing Emergency Medical Center

\section{Fanlin Zhou}

Chongqing Universtiy Cancer hospital

\section{Jie Liang}

Third Military Medical University Southwest Hospital Department of Pathology

\section{Wuquan Deng}

Chongqing Emergency Medical Center

\section{Shengbing Li}

The Second hospital of Chongqing medical University

\section{Wenwen Chen}

The Second hospital of Chongqing medical University

Ke Li

The Second hospital of Chongqing medical University

\section{Yu Li}

Chongqing University Cancer hospital

\section{Dongfang Liu}

The Second hospital of Chongqing medical University

\section{Gangyi Yang}

Peking University People's Hospital

Mingyuan Tian ( $\square$ mingyuant@hospital.cqmu.edu.cn )

The Second hospital of Chongqing medical University https://orcid.org/0000-0002-4645-3625

\section{Research}

Keywords: sitagliptin, gut microbiome, type 2 diabetes mellitus, central nervous system, microbiota-gutbrain axis, $16 \mathrm{~S}$ rRNA sequencing, hippocampus 
Posted Date: January 27th, 2020

DOl: https://doi.org/10.21203/rs.2.21932/v1

License: (c) (i) This work is licensed under a Creative Commons Attribution 4.0 International License. Read Full License 


\section{Abstract}

Background: Type 2 Diabetes mellitus is characterized by impaired glucose metabolism and insulin resistance with increasing risk of specific complication, including central neuropathy. Dipeptidyl peptidase 4 (DPP-4) inhibitors are agents designed to regulate hyperglicemia through increasing the halflife of incretins. Although they are administered orally, their effects on central neuropathy and gut microbiota remain unclear. Our objective was aimed to identify the impact of the DPP-4 inhibitor sitagliptin on gut microbiota and central neuropathy in a rat model of streptozotocin (STZ)-induced diabetes Methods: Diabetic rats were induced by a high-fat diet and streptozotocin injection. Diabetic rats were orally administered a dose of sitagliptin $(10 \mathrm{mg} / \mathrm{kd} / \mathrm{d})$, and normal diet for 12 weeks. Fecal DNA extraction and sequencing based on analysis of 16S rRNA genes was used to determine the overall structure of microbiota in fecal samples. Then rats were killed, of which the brain tissue sections were observed for changes of neurons in the hippocampus by HE staining. Results: Sequencing of the V3-V4 regions of $16 \mathrm{~S}$ rRNA genes revealed that sitagliptin significantly altered the gut microbiota. Compared to the diabetic group, Shannon index and the abundance of phylum Bacteroidetes decreased significantly, whereas the proportion of phylum Firmicutes increased significantly in the sitagliptin-treated rats. Moreover, higher abundance of a few putative short-chain fatty acid-producing bacteria, including Clostridium and Fusobacterium was observed remarkably after sitaglitin treatment. Genera Lactobacillus , Streptococcus and Clostridium, which could produce some neurotransmitters, were also found increased substantially in the sitagliptin-treated rats. Notably, we found that sitagliptin treatment could ameliorate the abnormal changes of pyramidal neurons in hippocampus with HE staining. Conclusions: Our study demonstrated that sitagliptin prevented the neuronal damage of hippocampus in type 2 diabetic rats, accompanied by changes of gut microbiome. It indicated that sitagliptin might alleviate diabetic central neuropathy through triggering "microbiota-gut-brain" axis.

\section{Introduction}

Type 2 diabetes mellitus is a chronic disorder characterized by disrupt glucose metabolism with increasing risk of specific complications, including vascular pathology, retinopathy, central neuropathy and so on. Accumulating researches have been paid attentions on central neuropathy, which refers to neuron damage and may eventually result in cognitive impairment [1]. It was originally postulated that diabetic central neuropathy occurs due to the influence of hyperglycemia on structural or functional domain of brain [2]. Hyperglycemia may damage neuron growth and maturation and increase amylin production, which may result in AD (Alzheimer disease) [2]. And a recent study has shown that glycemic variability is a key underlying reasons for diabetic central neuropathy [3]. However, ample evidences support that the microbiota's role in the pathology of type 2 diabetes [4, 5], and gut SCFA-producing bacteria regulate hippocampal neurogenesis [6], which challenge the originally postulated underlying mechanism. Specifically, chronic broad-spectrum combinatorial antibiotic treatment attenuates neurodegenerative pathology in murine model of $A D$ [7]. Additionally, it is well known that insulin resistance is a main feature of type 2 diabetes, and regarded as a potentially critical feature of $A D$ and 
related dementias [8]. These findings demonstrate that the underlying mechanism for diabetic central neuropathy remains unclear.

The gastrointestinal tract consists of trillions of bacteria collectively termed as gut microbiota, which interact with the host by immune, neuroendocrine and neural pathways. These pathways are regarded as the microbiota-gut-brain axis [9]. There is accumulating evidence that gut microbiota modulate brain development, function and behavior through the axis. In recent experiments, transplantation of fecal samples pooled from patients with ASD (autism spectrum disorder) into germ-free mice resulted in autistic behaviors with alternative splicing of ASD-associated genes in brains [10]. Modulation of microbiota is increasingly being regarded as an promising target for the development of new potential intervention for a wide variety of nervous disorders including depression, PD (Parkinson's desease) [11] and $A D$ [12].

Enteroendocrine cells in intestinal epithelium release incretins followed by nutrient ingestion. One of main incretins-glucagon-like peptide (GLP) 1 increase the postprandial secretion of insulin and regulate glucose level [13]. However its short half-life span limited GLP-1 activities, for it is rapidly cleaved and inactivated by a membrane-anchored cell surface peptidase named dipeptidyl peptidase-4 (DPP-4).

Sitagliptin, a novel type of DPP-4 inhibitor, has been widely used to treat type 2 diabetes via preventing the inactivation of GLP-1. However, Waget A et al. [14] reported that sitagliptin could regulate glucose level by triggering the gut-brain axis other than local inhibition of intestinal DPP-4 activity. Furthermore, sitagliptin treatment substantially changed the structure of gut microbiota in STZ-induced type 2 diabetic rats [15]. Additionally, recent studies have shown that sitagliptin could improve cognitive function in type 2 diabetic patients with and without Alzheimer's disease [16] and in insulin-resistant rats [17]. It has been found that sitagliptin enhanced hippocampal neurogenesis and attenuated cognitive impairment by up regulating some key genes linked to recognition memory in high-fat-fed mice [18]. However, it is currently unknown whether structure of microbiota and diabetic central neuropathy could be regulated by sitagliptin treatment.

Given that microbiota-gut-brain axis has been a potential target for the treatment of central nervous disease. We here investigated for the first time, using MiSeq sequencing, the effect of sitagliptin on the gut microbiota and central neuropathy in type 2 diabetic rats.

\section{Material And Methods}

\section{Generation of diabetic rats and drug treatment}

Five-week-old male Sprague-Dawley rats were acquired from the experimental animal center of Chongqing Medical University (Chongqing, China). All rats were caged at $24 \pm 1{ }^{\circ} \mathrm{C}$ with lights on from 6:00 a.m. to 6:00 p.m. 
The rats were fed on a standard diet (kcal \%: 10\% fat, 20\% protein, and 70\% carbohydrate; $3.85 \mathrm{kcal} / \mathrm{gm}$ ) or a high-fat diet (kcal \%: 45\% fat, 20\% protein, and 35\% carbohydrate; 4.73 kcal/gm, Research Diet, Medicine Ltd, Jiangsu, China). After exposure to the respective diets for 4 weeks, the rats were injected intraperitoneally with streptozocin ( $70 \mathrm{mg} / \mathrm{kg}$ body weight) or saline after being deprived of food for $10 \mathrm{~h}$, and then, they continued with their original diet through the whole experimentation period. Fasting blood glucose $>16.0 \mathrm{mmol} / \mathrm{L}$ was determined to be the standard definition for the type 2 diabetes model at 3rd day after streptozocin treatment.

The diabetic rats were randomly divided into two subgroups: T2DM treated with vehicle only (T2DM, $n=$ 9), T2DM treated with $10 \mathrm{mg}$ sitagliptin/kg body weight/day (T2DM-Sit, $n=9$ ). The normal diet-fed rats were orally administered vehicle only (ND, $n=6)$ or siltagliptin (10 mg/kg/day) (ND-Sit, $n=5)$. After 12 weeks of treatment, fresh stools were collected by stimulating the anus in the ND, ND-Sit, T2DM and T2DM-Sit groups and then immediately stored at $-80^{\circ} \mathrm{C}$ for subsequent analysis.

\section{Fecal DNA Extraction And Sequencing}

Total DNA was extracted from frozen feces by using a commercially available kit (QIAamp Fast DNA Stool Mini Kit; Qiagen) according to the manufacturer's instructions. The 16S rRNA genes were amplified using the 341F/806R primer set targeting the V3-V4 region (for 341F 5'-CCTACGGGNGGCWGCAG-3'; for 806R, 5'-GACTACHVGGGTATCTAATCC-3'). Purification of the amplified PCR products was conducted by using a QIAquick PCR purification kit. Equimolar amounts of purified PCR products were pooled and processed for sequencing. And DNA sequencing was performed on an Illumina MiSeq instrument with barcoding using a sequence kit (version 3.0) for optimal pair-end sequence reads.

FastQC (http://www.bioinformatics.babraham.ac.uk/projects/fastqc/) was firstly used to evaluate the quality of sequencing data. After removed the Phix sequence, the selected high quality sequences were further processed with Mothur software [19]. Paired end reads were merged into tags, either tags with high amount of ambiguous bases and homo-polymers, or tags out of the expected range were removed. Then tags were aligned to SILVA 119 [20] 16S rRNA gene sequences to keep the tags with correct alignment region and coordinates. A pre-clustering algorithm was utilized for further denoising and the chimeric sequences were discarded based on prediction by UCHIME [21]. Substantial taxonomic ranks were assigned by using Ribosomal Database Project (RDP) Naive Bayesian Classifier [22] trained on the RDP 16S rRNA gene training set (version 10) with an $80 \%$ pseudo-bootstrap confidence score. Sequences that either were not classified to the level of kingdom, or classified as Archaea, Eukaryota, chloroplasts, or mitochondria were culled. Finally, sequences were split into groups corresponding to their taxonomy and assigned to operational taxonomic units (OTUs) at 97\% similarity level.

\section{HE Staining}


All rats were anesthetized by an intraperitoneal injection of $10 \%$ chloral hydrate and euthanized. Then rats were perfused with $4 \%$ paraformaldehyde in PBS. Immediately, brains were rapidly removed and fixed in formaldehyde buffer. For morphological analysis, brains were cryoprotected for two days in $30 \%$ sucrose solution and sectioned. $5 \mu \mathrm{m}$ thick coronal sections were obtained by using a crvostas (Leica, Germany). For HE staining, the sections were stained with hematoxylin and eosin, dehydrated in 95\%, $90 \%$ and $70 \%$ ethanol, cleared in xylene, mounted in neutral gum.

\section{Data Analysis}

Continuous variables were characterized as means \pm standard deviations, while categorical data were reported as ratios or percentages. Unpaired t-tests were used to study differences in continuous variables and $\chi 2$ tests were used to analyze categorical variables using $R$ software with $P$ values of less than 0.05 . Nonmetric multidimensional scaling (NMDS) analysis was performed using vegan package in $\mathrm{R}$ software.

The LEfSe (Linear Discriminant Analysis Effect Size) algorithm [23] was used to identify taxa with differentiating relative abundance between two different groups with the logarithmic LDA score at 2.0.

\section{Results}

\section{Sitagliptin reduces the histopatholgical changes induced by diabetes}

As shown in Figure 1, pyramidal neurons in the hippocampus of the ND group and ND-Sit groups were intact, with round and full nuclei and clear nucleolus. However, in the T2DM group, the neurons have many morphological changes, including swelling of neurons, shrunken neurons with darkly stained condensed nuclei and a disordered arrangement and irregular pyramidal layer. After sitagliptin treatment in T2DM-Sit group, the pathological changes were improved. These results suggest that sitagliptin can alleviate neuron damage caused by type 2 dibetes mellitus.

\section{Difference on gut microbial diversity in response to sitagliptin treatment}

The Shannon index was applied to evaluate microbial diversity. Average value of Shannon index was $7.12 \pm 1.85,5.76 \pm 1.13,7.63 \pm 1.61$ and $6.33 \pm 1.28$ for ND, ND-Sit, T2DM and T2DM-Sit groups respectively. Further statistical analysis showed that T2DM group had almost similar microbial diversity with the ND group $(P=0.59)$, while sitagliptin treatment reduced the microbial diversity in the ND-Sit $(P=$ $0.17)$ and T2DM-Sit group $(P=0.077)$ (Figure 2A). 
NMDS analysis was performed on the relative taxa abundance of the gut microbial communities, a significantly distinct separation in the beta diversity of the gut microbial communities among the ND-Sit, T2DM and T2DM-Sit groups was observed. While the ND and T2DM-Sit groups showed a large part of overlaps (Figure 2B). It revealed that the gut microbiota structure changed significantly in response to high-fat diet feeding and sitagliptin administration.

\section{Microbial structure among different conditions}

To characterize the composition of the gut microbiota, a total of 2,551,356 se/quencing reads (an average of 87,978 sequences per sample) were obtained from 29 samples. The overall gut microbiota composition of each sample at the phylum level was shown in Figure 3A. Figure 3B showed the average composition at the phylum level. It demonstrated that Firmicutes and Bacteroidetes were the most abundant in all enrolled subjects, and they accounted for the majority of microflora ( $>80 \%)$ in each condition. The relative abundance of Firmicutes in T2DM group significantly decreased compared to that in ND group (24.15\% versus $38.95 \%, P=0.04)$, while the relative abundance of Bacteroidetes presented an increase trend $(57.81 \%$ versus $44.97 \%, P=0.15)$. The relative abundance of Firmicutes in T2D-Sit group was significantly more than that in T2DM group ( $36.87 \%$ versus $24.15 \%, p=0.03$ ), whereas the relative abundance of Bacteroidetes decreased dramatically ( $45.0 \%$ versus $57.81 \%, P=0.03)$. Compared to the normal rats, the microbiota in the diabetic rats featured a higher percentage of Bacteroidetes, a lower percentage of Firmicutes and a higher ratio of Bacteroidetes: Firmicutes. But this trend was reversed after sitagliptin treatment, which was shown both in T2DM-Sit and ND-Sit groups. Sitagliptin treatment also increased the relative abundance of Proteobacteria and Candidatus_Saccharibacteria (Figure 3B).

The main family was Enterobacteriaceae, Lachnospiraceae, Lactobacillaceae, Peptostreptococcaceae, Porphyromonadaceae, Prevotellaceae, Ruminococcaceae, Spirochaetaceae and Streptococcaceae (Figure 3C). It was noteworthy that the relative abundance of Prevotellaceae in the diabetic rats increased compared to the normal rats $(28.09 \%$ versus $20.73 \%)$, but decreased after sitagliptin treatment $(23.08 \%$ versus $28.09 \%$ ). Similar changes were observed at the family of Porphyromonadaceae and Spirochaetaceae. However, compared to the normal condition, the relative abundance of Ruminococcaceae decreased in the diabetic condition (8.17\% versus $6.09 \%)$, but increased after sitagliptin treatment (6.09\% versus $6.87 \%)$, and family Streptococcaceae presented a similar trend.

\section{Differences of structure changes in the gut microbiota in response to sitagliptin treatment}

There were significant alterations in the structure of intestinal bacteria among the ND, T2DM, and T2DMSit groups. LEfSe analysis was performed to investigate differences in the community composition between ND and T2DM groups, T2DM and T2DM-Sit groups. The greatest differences in taxa were 
presented in Figure 3. Compared to the normal rats, genera Allobaculum, Bifidobacterium, Anaerobacter increased and genera Bilophila, Fusobacterium, Campylobacteriales, Aerococcus, Romboutsia and Paraprevotella decreased in the diabetic condition (Figure 4A). Under sitagliptin treatment, 16 genera were key variables that separated the gut microbiota, among them 10 genera increased and 6 genera decreased, compared to the T2D group. The enriched genera were Lactobacillus, Streptococcus, Clostridium_IV, Fusobacterium, Clostridium_XIVb, Parasporobacterium, Enhydrobacter, Barnesiella, Aerococcus and Collinsella. The decreased genera were Falsiporphyromonas, Mucispirillum, Eisenbergiella, Oligosphaera, Allobaculum, and Alloprevotella (Figure 4B).

\section{Discussion}

Diabetic central neuropathy, which refers to neuron damage and may eventually result in dementia, is one of the most common complications of type 2 diabetes. Sitagliptin has been widely prescribed as pharmacotherapy for the treatment of patients with type 2 diabetes. However, the effects of sitagliptin on diabetic central neuropathy and intestinal ecosystem remain poorly studied. Our work is the first to consider that sitagliptin ameliorates diabetic central neuropathy via preventing the hippocampal neuronal damage and alters structure of gut microbiota in type 2 diabetic rats. Our current study demonstrates that sitagliptin might alleviate diabetic central neuropathy through triggering microbiota-gut-brain axis.

Diabetic central neuropathy refers to neuronal damage and brain physiological and structural changes caused by diabetes. Accumulating evidences indicated that diabetes is highly connected with a progressive cognitive impairment and results in $A D$ [24]. Hippocampus is responsible for cognitive function. Some researches have shown that type 2 diabetes results in hippocampal neuron apoptosis by Estrogen receptors-PI3/Akt pathway [25]. Kim Ms et al. [26] demonstrated that microbial dysbiosis contribute to the pathogenesis of Alzheimer's disease related to cognitive impairment and fecal microbiota transfer reversed the AD pathophysiology. Meanwhile, dysbiosis of microbiota played an important role in the pathological process of diabetic cognition impairment [27]. The underlying mechanism needs to be explored.

Sitagliptin is currently the common treatment for type 2 diabetes through inhibiting DPP-4 activity. We observed that sitagliptin alleviated hippocampal neuron loss induced by type 2 diabetes in rats. As forementioned, microbiota affects process of $A D$ and diabetic cognition impairment. Therefore, the relationships between sitagliptin and diabetic central neuropathy, as well as microbiota remain unclear.

Type 2 diabetes is characterized by elevated glucose levels due partly to insulin resistance and insufficient secretion of insulin, with increased prevalence at an alarming rate. Genetic and environmental factors, and other risk factors containing age, dietary habits, obesity and lifestyle, have been confirmed to account for the disease. Some studies observed that dysbiosis of the human gut microbiota is connected with type 2 diabetes. Qin [28] found type 2 diabetes patients had gut bacterial dysbiosis with a decrease in butyrate-producing bacteria, which have been confirmed to alleviate several diseases, and a rise in 
some opportunistic pathogens such as Escherichia coli in Chinese population. Similar results were observed in European type 2 diabetes patients [29]. Similarly, some researches found that gut microbiota were substantially altered in STZ-induced type 2 diabetic rats $[15,30]$.

The gut microbiota comprising the collective genome of 100 trillion microorganisms in the gastrointestinal tract is regarded as a major player affecting the health status of the host. Intestinal microbes influence the central nervous system through central, autonomic and enteroendocrine nervous system, production of various metabolites as well as microbial-associated-molecular patterns (MAMPs) produced by microbiota, the bidirectional communication has been suggested a microbiota-gut-brain axis [9]. Accumulating evidences showed that disorder of gut microbiota involved in the development and function of central nervous system. Some studies showed that dysbiosis of microbiota was associated with cognitive and behavioral dysfunction in mice [31,32]. Microbiota-gut-brain axis is regarding as a recognized targeted strategy for the prevention and treatment of central nervous system related diseases $[33,34]$.

Our objective was to determine whether sitagliptin treatment played an important role in returning the gut microbiota back to the normal. Moreover, the sitagliptin-treated rats displayed changed microbial diversity compared to diabetic rats, which was in accordance with the previous study [35]. The data showed that the rat gut microbiome was largely dominated by phyla Firmicutes and Bacteroidetes. Compared to normal rats, diabetic rats related to a significant shift in the gut microbiota, with a reduced percentage of Firmicutes and Candidatus_Saccharibacteria and an increased proportion of phyla Bacteroidetes and Spirochaetes. In addition, the Bacteroidetes: Firmicutes ratio also increased in the T2DM group, similar to previous studies [36]. Furthermore, sitagliptin treatment normalized the ratio of Bacteroidetes: Firmicutes, with an increase in abundance of Firmicutes and a decrease of Bacteroidetes, contradicting the previous findings with higher proportion of Bacteroidetes and lower proportion of Firmicutes after sitagliptin treatment [15]. The main reason for the inconsistency was the limitation of small sample size, which should be confirmed further with larger size of samples.

Enterobacteriaceae, Lactobacillacea, Prevotellaceae, and Ruminococcaceae were the main families of all the rats. As a part of the phylum Bacteroidetes, family Prevotellaceae has some genera associated with an increase risk of diabetes mellitus [37]. It was more abundant in the diabetic rats compared to the normal rats, decreased in the sitagliptin-treated rats in this study. Among phylum Firmicutes, family Ruminococcaceae was a major utilizer of plant polysaccharides [38, 39], and its enrichment might counteract the development of autoimmune diabetes [40]. The result showed that a higher abundance of Ruminococcaceae in the sitagliptin-treated rats than in the diabetic rats. Notably, family Lactobacillaceae increased significantly after sitagliptin treatment compared to the T2DM group, one of its main genera, Lactobacillus also presented a higher abundance in the T2DM-Sit group according to the LEfSe analysis. The mechanism might be related that some Lactobacillus species could metabolize tryptophan into indole-3-aldyhyde, which acts on the aryl hydrocarbon receptor (AhR) in intestinal immune cells [41]. The ligand for AhR was indole, which could trigger the secretion of glucagon-like peptide -1 in intestinal L cells [41]. 
The LEfSe analysis showed that the diabetic rats with an enrichment of family Enterobacteriaceae and Enterococcaceae, order Enterobacteriales. As potential pro-inflammatory microorganism in the gut, Enterobacteriaceae and Enterococcaceae both presented an increase in the T2DM patients [42]. They could further contribute to the rise of imflammatory level in the host, supporting the evolution of insulin resistance [43]. Previous study also observed a higher abundance of the gram-negative bacteria Enterobacteriales in the diabetic condition, which may be mechanistically linked to increased colonic permeability irrespective of glucose tolerance status [44].

In our study, genus Lactobacillus, genera Streptococcus, Clostridium and Fusobacterium increased significantly compared to diabetic group after sitagliptin treatment. They can produce a range of neurotransmitters to participate in the regulation of varied and important physiological processes, including immunomodulation, adiposity, and energy balance [41], genus Streptococcus produce serotonin [45]. Lactobacillus produces acetylcholine and $y$-aminobutyric acid [46]. Species Clostridium sporogens of genus Clostridium metabolizes tryptophan into indole and subsequently 3-indolepropionic acid, a highly potent neuroprotective antioxidant [47]. Moreover, Clostridium and Fusobacterium are SCFAsproducing bacterium mainly exists in cecum and colon [48].

Short-chain fatty acids (SCFAs) including acetic acid, butyric acid and propionic acid are major metabolic and products of gut microbial degradation of dietary fiber in the colon [49]. SCFAs are ligands for G protein-coupled receptor (GPCR) GPR41 and induce secretion of the enteroendocrine hormone peptide YY (PYY) from gut epithelial L-cells, which restrains gut motility and add energy harvest from the diet [50]. Accumulating evidence showed that SCFA-GPR interactions would allow direct signaling from gut to the central nervous system. The maturation and function of microglia, which are the resident macrophages of the brain, were dependent on gut microbiota, and SCFAs and GPR were necessary to maintain microglia homeostasis and integrity of the blood-brain barrier in mice [51, 52]. Recently, a study showed that high-fructose diet-induced gut dysbiosis with reduced SCFA in the impairment of colonic epithelia barrier that resulted in hippocampal neuroinflammation and neuronal loss in mice, and the neurodegenerative changes could be prevented by SCFA treatment [53]. These results further suggest that SCFAs exert protective effects on modulation of neurological function. Which may help to explain partly the neuroprotective effects of sitagliptin on hippocamlal neuron loss induced by type 2 diabetes by triggering microbiota-gut-brain axis.

However, lack of the proportions of SCFAs and neurotransmitters existed in this study, which made the changes of SCFAs after sitagliptin treatment without data support. Therefore in the further study, the quantification of these metabolites should be taken into account. Additionally, there are some issues that still need to be resolved: detection of microbial interaction and discrepancy at lower taxonomic levels; large cohort studies to confirm these findings; longitudinal studies to track the gut microbiome and its influences on CNS function.

\section{Conclusion}


In conclusion, the findings suggested that sitagliptin treatment enriched Firmicutes and some SCFAproducing bacteria and reduced gut microbial diversity for eventually mediated their beneficial effects on the host. Additionally, it attenuated significantly hippocampal neuron loss induced by type 2 diabetes. That modifying the specific structure and characteristics of the gut microbiota by sitagliptin treatment might become a therapeutic strategy for diabetic central neuropathy.

\section{List Of Abbreviations}

DPP-4, Dipeptidyl peptidase 4; STZ, streptozotocin; AD, Alzheimer disease; ASD, autism spectrum disorder; PD,Parkinson's desease; GLP-1,glucagon-like peptide 1; NMDS, multidimensional scaling ;ND,Normal diet; T2DM, Type 2 diabetes mellitus; ND-Sit, Normal diet-Sitagliptin; T2DM-Sit, Type 2 diabetes mellitusSitagliptin; MAMPs,associated-molecular patterns ; SCFAs,Short-chain fatty acids; GPCR,G proteincoupled receptor; PYY,hormone peptide YY; CNS,Central nervous system.

\section{Declarations}

\section{Ethics approval and consent to participate}

All experiments on animals were approved by the Chongqing Medical University Institutional Animal Care and Use Committee (animal certificate number:SCXF2012-0001)

\section{Consent for publication}

Not applicable

\section{Availability of data and materials}

The datasets generated and analyzed during the current study are available in the NCBI Sequence Read Archive (BioProjectID PRJNA601240).

\section{Competing interests}

The authors declare that they have no competing interests.

\section{Author's contributions}

Y.C, T.X., F.Z and J.L.conceived and carried out the experiments.H.W, and W.D analyzed data. Y.C.,T.X.and M.T drafted the manuscript. S.L.,W.C and K.L made the Figure.Y.L.,D.L. and G.Y. revise and edit the manuscript. M.T. is the guarantor of this work and, as such, had full access to all the data in the study and takes responsibility for the integrity of the data and the accuracy of the data analysis. All authors were involved in writing the paper and had final approval of the submitted and published versions. 


\section{Funding}

This work was supported by research grants from the National Natural Science Foundation of China (81801389)

\section{Acknowledgements}

We thank Professor Yu Li and Gangyi Yang for giving the Chance to learn Alzheimer disease and diabetes respectively,Thanks for their helps and directions these years. We thank Professor Dongfang Liu for her support and encouragement.The authors are grateful to Bin Xiao for technical assistance. We also thank the committee of European Association for the Study of Diabetes for giving an opportunity to attend the 55th EASD Annual Meeting.

\section{References}

1. Simo R, Ciudin A, Simo-Servat O, Hernández C et al. Cognitive impairment and dementia: a new emerging complication of type 2 diabetes-The diabetologist's perspective. Acta Diabetol. 2017;54:417-24.

2. Malone Jl. Diabetic central neuropathy: CNS damage related to hyperglycemia. Diabetes. 2016;65:355-7.

3. Yang J, Zhao Z, Yuan H, Ma X, Li Y, Wang H, et al. The mechanisms of glycemic variability accelerate diabetic central neuropathy and diabetic peripheral neuropathy in diabetic rats. Biochem Biophys Res Commun. 2019;510:35-41.

4. Thingholm LB, Ruhlemann MC, Koch M, Fuqua B, Laucke G, Boehm R, et al. Obese individuals with and without type 2 diabetes show different gut microbial functional capacity and composition. Cell Host Microbe. 2019;26(2):252-64.

5. Sanna S, van Zuydam NR, Mahajan A, Kurilshikov A, Vich Vila A, Vosa U, et al. Causal relationships among the gut microbiome, short-chain fatty acids and metabolic diseases. Nat Genet. 2019;51(4):600-5.

6. Mohle L, Mattei D, Heimesaat MM, Bereswill S, Fischer A, Alutis M, et al. Ly6C(hi) monocytes provide a link between antibiotic-induced changes in gut microbiota and adult hippocampal neurogenesis. Cell Rep. 2016;15:1945-56.

7. Minter MR, Hinterleitner R, Meisel M, Zhang C, Leone V, Zhang X, et al. Antibiotic-induced perturbations in microbial diversity during post-natal development alters amyloid pathology in an aged APPSWE/PS1DeltaE9 murine model of Alzheimer's disease. Sci Rep. 2017;7:10411.

8. Arnold SE, Arvanitakis Z, Macauley-Rambach SL, Koenig AM, Wang HY, Ahima RS, et al. Brain insulin resistance in type 2 diabetes and Alzheimer disease: concepts and conundrums. Nat Rev Neurol. 2018;14(3):168-81.

9. Cryan JF, O'Riordan KJ, Cowan CSM, Sandhu KV, Bastiaanssen TFS, Boehme M, et al. The microbiota-gut-brain axis. Physiol Rev. 2019;99(4):1877-2013. 
10. Sharon G, Cruz NJ, Kang DW, Gandal MJ, Wang B, Kim YM, et al. Human gut microbiota from autism spectrum disorder promote behavioral symptoms in mice. Cell. 2019;177:1600-18.

11. Perez-Pardo P, Dodiya HB, Engen PA, Forsyth CB, Huschens AM, Shaikh M, et al. Role of TLR4 in the gut-brain axis in Parkinson's disease: a translational study from men to mice. Gut. 2019;68:829-43.

12. Castillo-Alvarez F, Marzo-Sola ME. Role of the gut microbiota in the development of various neurological diseases. Neurologia. 2019.

13. Seino Y, Fukushima M, Yabe D. GIP and GLP-1, the two incretin hormones: similarities and differences. J Diabetes Investig. 2010;1:8-23.

14. Waget A, Cabou C, Masseboeuf M, Cattan P, Armanet M, Karaca M, et al. Physiological and pharmacological mechanisms through which the DPP-4 inhibitor sitagliptin regulates glycemia in mice. Endocrinology. 2011;152:3018-29.

15. Yan X, Feng B, Li P, Tang Z, Wang L. Microflora disturbance during progression of glucose intolerance and effect of sitagliptin: an animal study. J Diabetes Res. 2016;2016:2093171.

16. Isik AT, Soysal P, Yay A, Usarel C. The effects of sitagliptin, a DPP-4 inhibitor, on cognitive functions in elderly diabetic patients with or without Alzheimer's disease. Diabetes Res Clin Pract. 2017;123:1928.

17. Pintana H, Apaijai N, Chattipakorn N, Chattipakorn SC. DPP-4 inhibitors improve cognition and brain mitochondrial function of insulin-resistant rats. J Endocrinol. 2013;218:1-11.

18. Gault VA, Lennox R, Flatt PR. Sitagliptin, a dipeptidyl peptidase-4 inhibitor, improves recognition memory, oxidative stress and hippocampal neurogenesis and upregulates key genes involved in cognitive decline. Diabetes Obes Metab. 2015;17:403-13.

19. Schloss PD, Westcott SL, Ryabin T, Hall JR, Hartmann M, Hollister EB, et al. Introducing mothur: opensource, platform-independent, community-supported software for describing and comparing microbial communities. Appl Environ Microbiol. 2009;75:7537-41.

20. Quast C, Pruesse E, Yilmaz P, Gerken J, Schweer T, Yarza P, et al. The SILVA ribosomal RNA gene database project: improved data processing and web-based tools. Nucleic Acids Res. 2013;41(Database issue):D590-6.

21. Edgar RC, Haas BJ, Clemente JC, Quince C, Knight R. UCHIME improves sensitivity and speed of chimera detection. Bioinformatics. 2011;27:2194-200.

22. Wang Q, Garrity GM, Tiedje JM, Cole JR. Naive Bayesian classifier for rapid assignment of rRNA sequences into the new bacterial taxonomy. Appl Environ Microbiol. 2007;73:5261-7.

23. Segata N, Izard J, Waldron L, Gevers D, Miropolsky L, Garrett WS, et al. Metagenomic biomarker discovery and explanation. Genome Biol. 2011;12:R60.

24. Koekkoek PS, Kappelle LJ, van den Berg E, Rutten GE, Biessels GJ. Cognitive function in patients with diabetes mellitus: guidance for daily care. Lancet Neurol. 2015;14:329-40.

25. Tang SS, Ren $Y$, Ren XQ, Cao JR, Hong H, Ji H, et al. ERa and/or ER $\beta$ activation ameliorates cognitive impairment, neurogenesis and apoptosis in type 2 diabetes mellitus mice. Exp Neurol. 2019;311:33- 
43.

26. Kim MS, Kim Y, Choi H, Kim W, Park S, Lee D, et al. Transfer of a healthy microbiota reduces amyloid and tau pathology in an Alzheimer's disease animal model. Gut. 2020;69:283-94.

27. Xu Y, Zhou H, Zhu Q. The impact of microbiota-gut-brain axis on diabetic cognition impairment. Front Aging Neurosci. 2017;9:106.

28. Qin J, Li Y, Cai Z, Li S, Zhu J, Zhang F, et al. A metagenome-wide association study of gut microbiota in type 2 diabetes. Nature. 2012;490:55-60.

29. Karlsson FH, Tremaroli V, Nookaew I, Bergström G, Behre CJ, Fageberg B, et al. Gut metagenome in European women with normal, impaired and diabetic glucose control. Nature. 2013;498:99-103.

30. Xiao S, Liu C, Chen M, Zou J, Zhang Z, Cui X, et al. Scutellariae radix and coptidis rhizoma ameliorate glycolipid metabolism of type 2 diabetic rats by modulating gut microbiota and its metabolites. Appl Microbiol Biotechnol. 2020;104:303-17.

31. Gareau MG, Wine E, Rodrigues DM, Cho JH, Whary MT, Philpott DJ, et al. Bacterial infection causes stress-induced memory dysfunction in mice. Gut. 2011;60:307-17.

32. Bruce-Keller AJ, Salbaum JM, Luo M, Blanchard E 4th, Taylor CM, Welsh DA, et al. Obese-type gut microbiota induce neurobehavioral changes in the absence of obesity. Biol Psychiatry. 2015;77:60715.

33. Raimondi MT, Albani D, Giordano C. An organ-on-a-chip engineered platform to study the microbiotagut-brain axis in neurodegeneration. Trends Mol Med. 2019;25:737-40.

34. Long-Smith C, O'Riordan KJ, Clarke G, Stanton C, Dinan TG, Cryan JF. Microbiota-Gut-Brain axis: new therapeutic opportunities. Annu Rev Pharmacol Toxicol. 2020;60: 477-502.

35. Zhang M, Feng R, Yang M, Qian C, Wang Z, Liu W, et al. Effects of metformin, acarbose, and sitagliptin monotherapy on gut microbiota in Zucker diabetic fatty rats. BMJ Open Diabetes Res Care. 2019,7:e000717.

36. Larsen N, Vogensen FK, van den Berg FW, Nielsen DS, Andreasen AS, Pedersen BK, et al. Gut microbiota in human adults with type 2 diabetes differs from non-diabetic adults. PLoS One. 2010;5:e9085.

37. Wei X, Tao J, Xiao S, Jiang S, Shang E, Zhu Z, et al. Xiexin Tang improves the symptom of type 2 diabetic rats by modulation of the gut microbiota. Sci Rep. 2018;8:3685.

38. Flint HJ, Scott KP, Duncan SH, Louis P, Forano E. Microbial degradation of complex carbohydrates in the gut. Gut Microbes. 2012;3:289-306.

39. Crost EH, Le Gall G, Laverde-Gomez JA, Mukhopadhya I, Fling HJ, Juge N. Mechanistic insights into the cross-feeding of Ruminococcus gnavus and Ruminococcus bromii on host and dietary carbohydrates. Front Microbiol. 2018;9:2558.

40. Toivonen RK, Emani R, Munukka E, Rintala A, Laiho A, Pietilä S, Pursiheimo JP, et al. Fermentable fibres condition colon microbiota and promote diabetogenesis in NOD mice. Diabetologia. 2014;57:2183-92. 
41. Zelante T, lannitti RG, Cunha C, De Luca A, Giovannini G, Pieraccini G, et al. Tryptophan catabolites from microbiota engage aryl hydrocarbon receptor and balance mucosal reactivity via interleukin-22. Immunity. 2013;39:372-85.

42. Candela M, Biagi E, Soverini M, Consolandi C, Quercia S, Severgnini M, et al. Modulation of gut microbiota dysbioses in type 2 diabetic patients by macrobiotic Ma-Pi 2 diet. Br J Nutr. 2016;116:8093.

43. Johnson AM, Olefsky JM. The origins and drivers of insulin resistance. Cell. 2013;152:673-84.

44. Pedersen C, ljaz UZ, Gallagher E, Horton F, Ellis RJ, Jaiyeola E, et al. Fecal Enterobacteriales enrichment is associated with increased in vivo intestinal permeability in humans. Physiol Rep. 2018;6:e13649.

45. Sarkar A, Lehto SM, Harty S, Dinan TG, Cryan JF, Burnet PWJ. Psychobiotics and the manipulation of bacteria-gut-brain signals. Trends Neurosci. 2016;39:763-81.

46. Dinan TG, Stilling RM, Stanton C, Cryan JF. Collective unconscious: how gut microbes shape human behavior. J Psychiatr Res. 2015;63:1-9.

47. Wikoff WR, Anfora AT, Liu J, Schultz PG, Lesley SA, Peters EC, et al. Metabolomics analysis reveals large effects of gut microflora on mammalian blood metabolites. Proce Natl Acad Sci USA. 2009;106:3698-703.

48. Hold GL, Schwiertz A, Aminov RI, Blaut M, Flint HJ. Oligonucleotide probes that detect quantitatively significant groups of butyrate-producing bacteria in human feces. Appl Environ Microbiol. 2003;69:4320-4.

49. Schroeder BO, Bäckhed F. Signals from the gut microbiota to distant organs in physiology and disease. Nat Med. 2016;22:1079-89.

50. Samuel BS, Shaito A, Motoike T, Rey FE, Backhed F, Manchester JK, et al. Effects of the gut microbiota on host adiposity are modulated by the short-chain fatty-acid binding $\mathrm{G}$ protein-coupled receptor, Gpr41. Proc Natl Acad Sci USA. 2008;105:16767-72.

51. Erny D, Hrabě de Angelis AL, Jaitin D, Wieghofer P, Staszewski O, David E, et al. Host microbiota constantly control maturation and function of microglia in the CNS. Nat Neurosci. 2015;18:965-77.

52. Braniste V, Al-Asmakh M, Kowal C, Anuar F, Abbaspour A, Tóth $M$, et al. The gut microbiota influences blood-brain barrier permeability in mice. Sci Transl Med. 2014;6:263ra158.

53. Li JM, Yu R, Zhang LP, Wen SY, Wang SJ, Zhang XY, et al. Dietary fructose-induced gut dysbiosis promotes mouse hippocampal neuroinflammation: a benefit of short-chain fatty acids. Microbiome. 2019;7:98.

\section{Figures}


ND

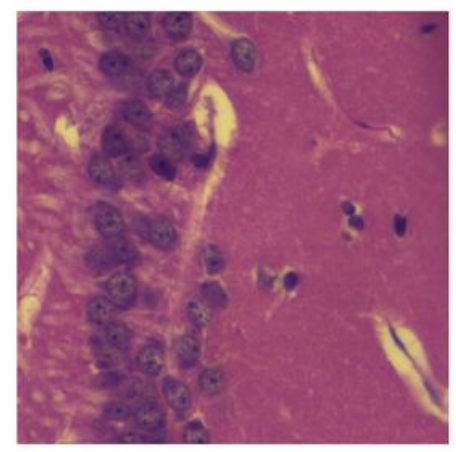

ND-Sit

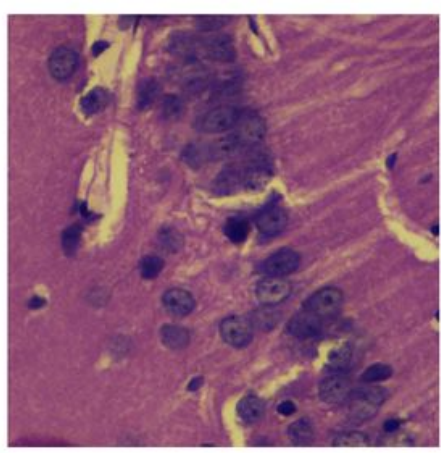

T2DM

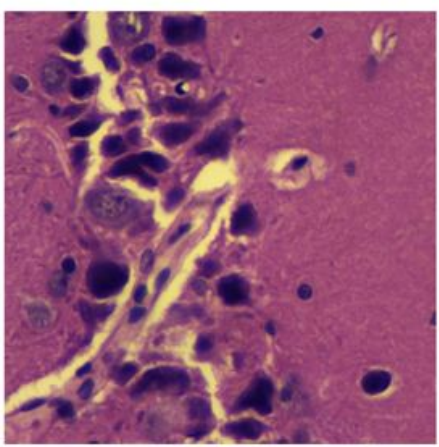

T2DM-Sit

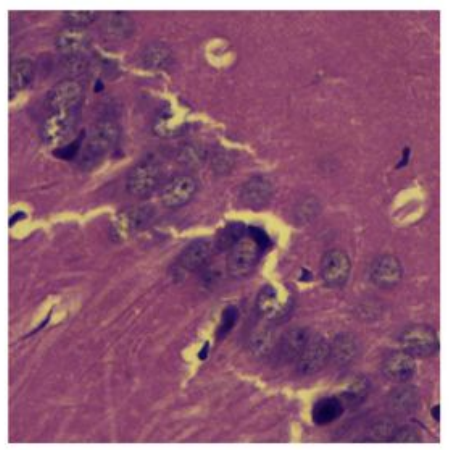

\section{Figure 1}

Morphological changes in pyramidal layer cells of hippocampus after sitagliptin treatment by HE staining.

A

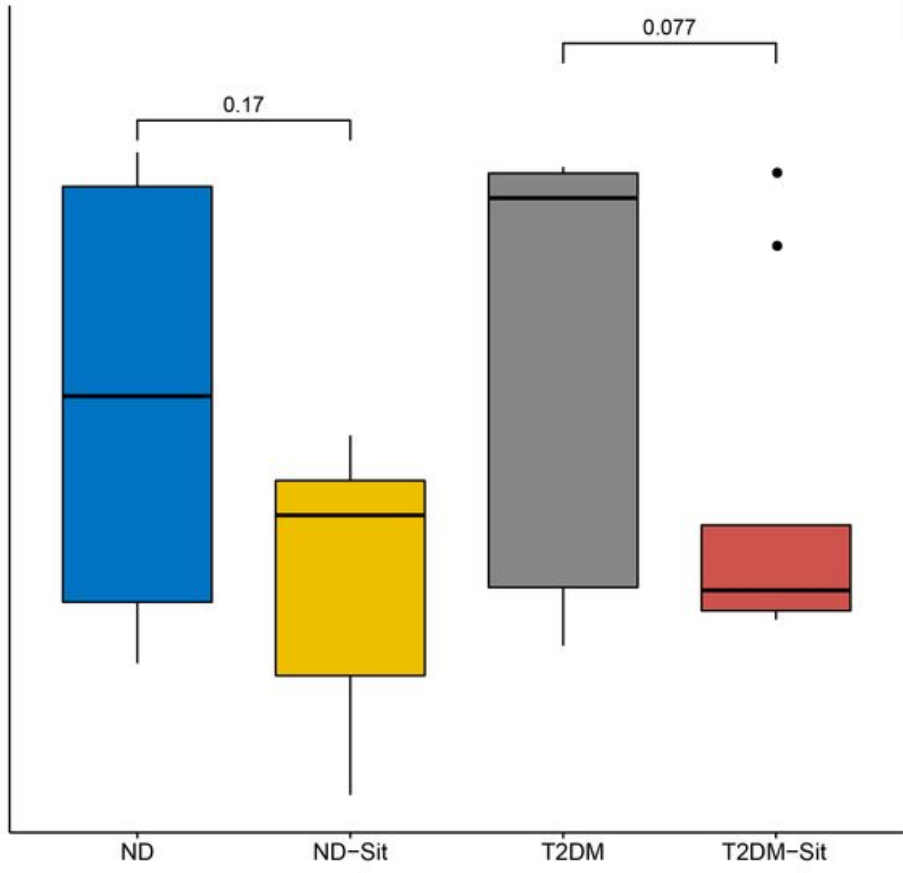

追 ND追ND-Sit追T2DM白 T2DM-Sit
B

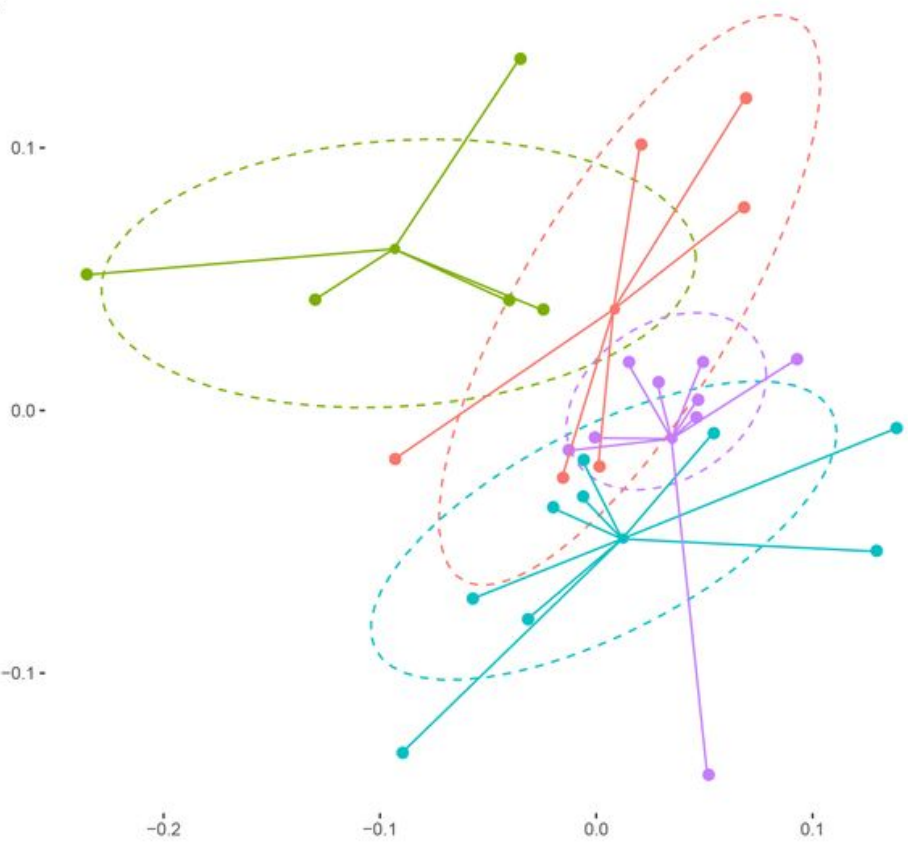

$\rightarrow N D \rightarrow N D-S i t \rightarrow-T 2 D M-T 2 D M-S i t$

\section{Figure 2}

Microbial biodiversity in response to sitagliptin treatment. (A) The Shannon index were shown as estimators. (B) NMDS analysis plots in intestinal microbiota based on OTU abundance. Each point represents the intestinal microbiota of a rat. 


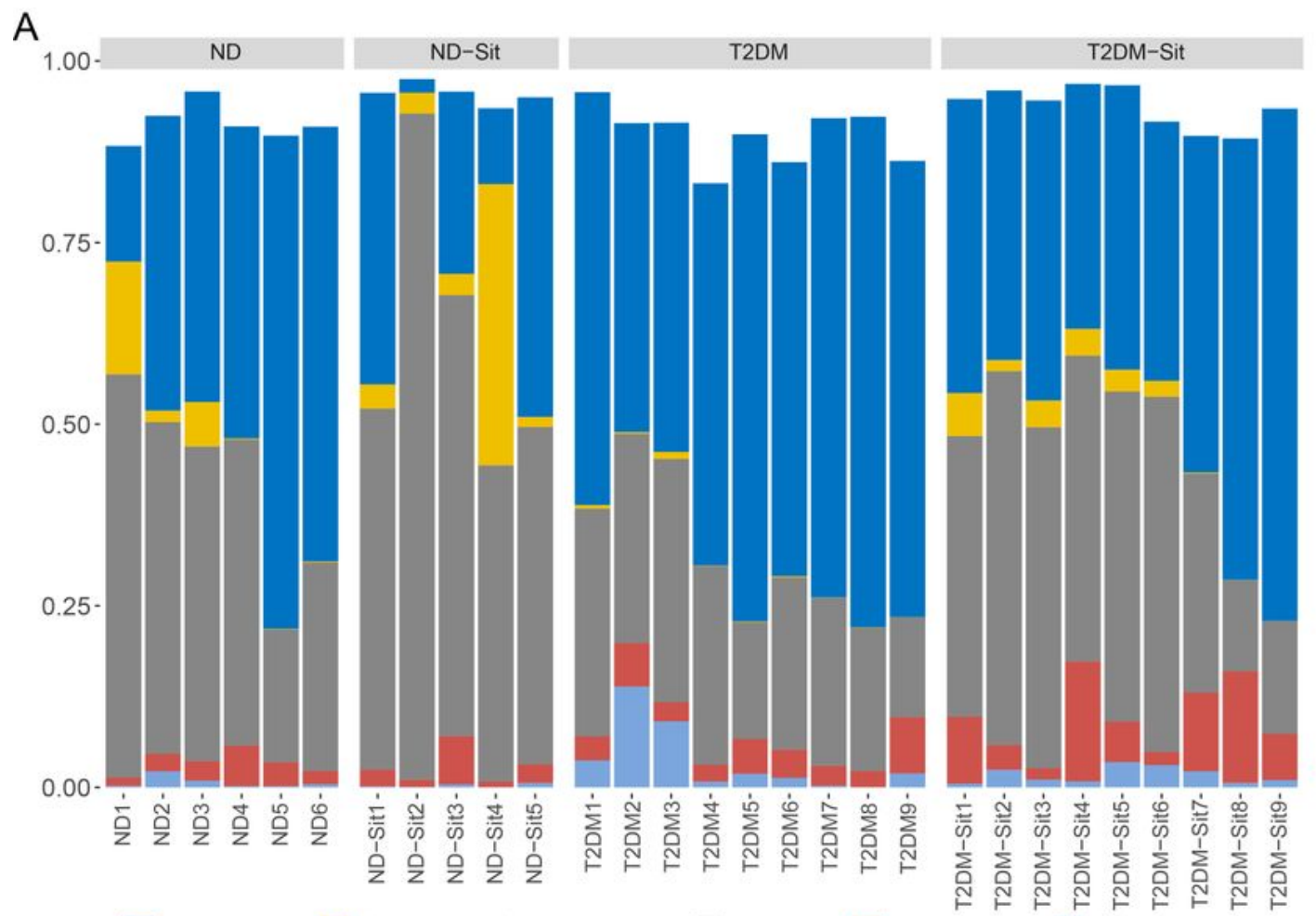

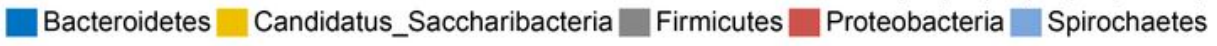

B

100 -

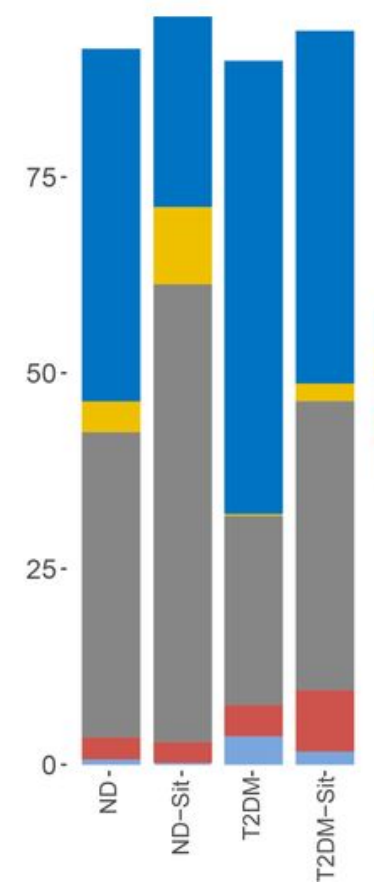

C

Candidatus_Saccharibacteria

Firmicutes

Proteobacteria

Spirochaetes
60 .

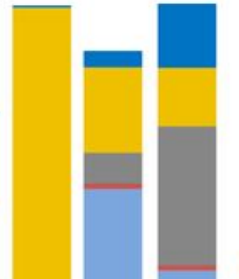

Enterobacteriaceae

Lachnospiraceae

Lactobacillaceae

Peptostreptococcaceae

Porphyromonadaceae

Prevotellaceae

Ruminococcaceae

Spirochaetaceae

Streptococcaceae

Figure 3

Composition of intestinal microbiome at different group. (A) Relative abundance at the phylum level of each subject. (B) Average relative abundance at the phylum level of ND, ND-Sit, T2DM and T2DM-Sit groups. (C) Average relative abundance at the family level of ND, ND-Sit, T2DM and T2DM-Sit groups. 
A

ND $\square$ T2DM

o Enterobacteriales

g Allobaculum

__Bifidobacteriales

g Bifidobacterium

$f$ Enterobacteriaceae

$f$ Enterococcaceae

Bifidobacteriaceae

g Anaerobacter

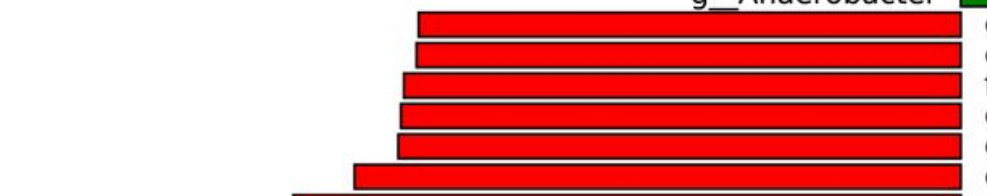

g Collinsella

c-Clostridia

f_Lactobacillaceae

g Bilophila

g-Fusobacterium

- Campylobacterales

g-Aerococcus

g_Romboutsia

g Paraprevotella

o_Clostridiales

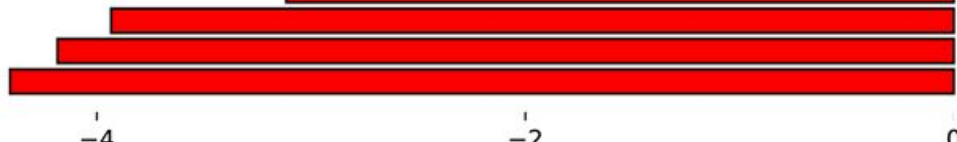

B

LDA SCORE $(\log 10)$

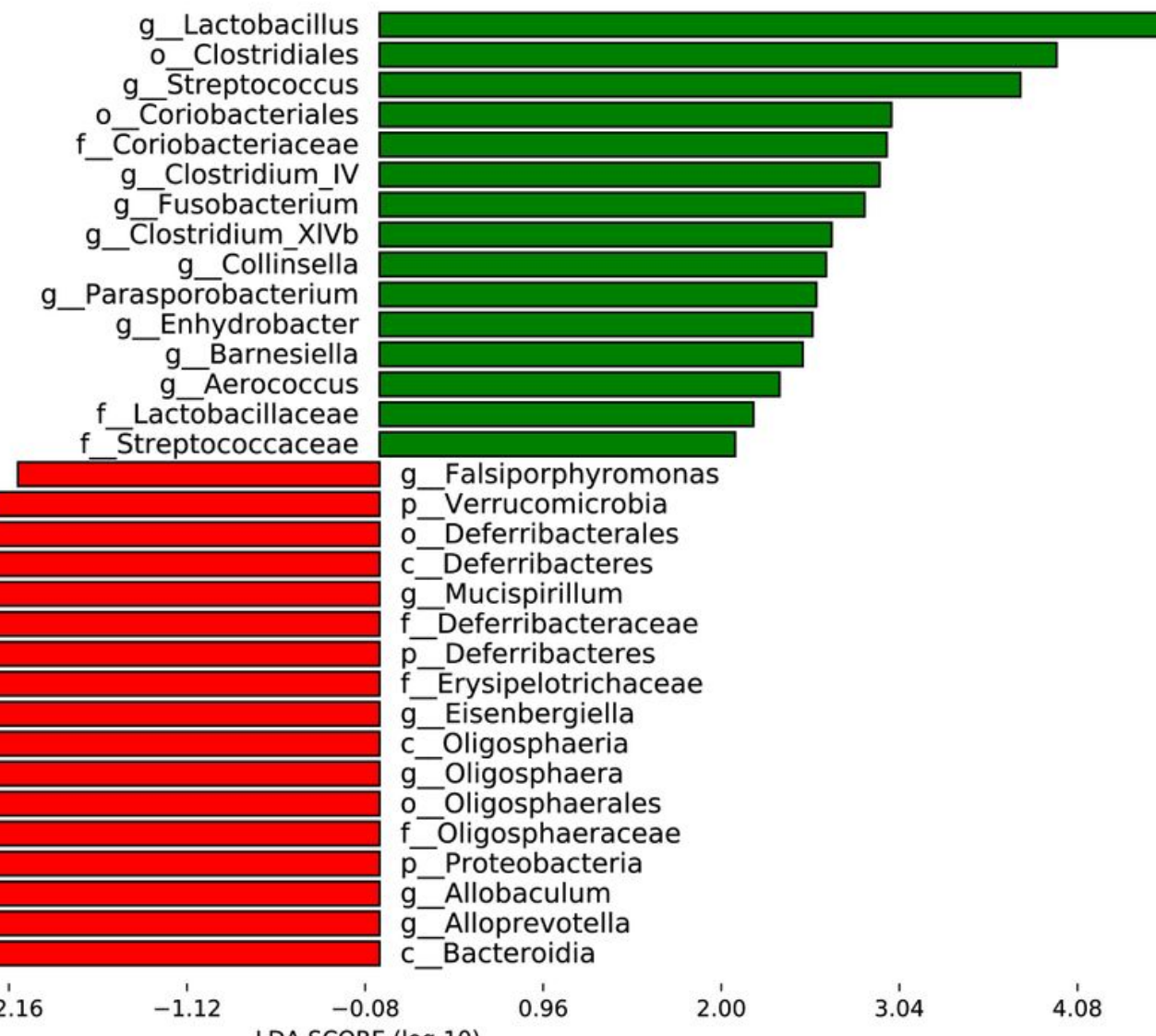

\section{Figure 4}

Histogram of the LDA scored for differentially abundant bacteria. LDA scores were calculated by LDA effect size using linear discriminant analysis. (A) Significant different bacteria between ND and T2DM groups. (B) Significant different bacteria between T2DM and T2DM-Sit groups. 